\title{
Modeling and Measurements of Electron Beam Scattering into Adjacent Particles
}

\author{
S.A. Wight and J.A. Small
}

Surface and Microanalysis Science Division, National Institute of Standards and Technology, 100 Bureau Drive, Gaithersburg, MD 20899-8371

Nanometer-sized particles and materials are in widespread use from personal use products to large scale industrial manufacturing. The health and environmental effects of exposure to these nanomaterials are not well known but the ability to accurately identify and characterize these materials is of the utmost importance.

Characterization of micrometer-sized particles by scanning electron microscopy and energy dispersive analysis has been widely studied over the last several decades. Accurate quantification of the chemical composition of micrometer sized particles is not without its difficulties and is often compared to the quantification of the similar material in the flat, polished bulk material [1]. Nanometer-sized particles offer their own set of analytical challenges. For example, is it better to analyze them in the SEM/probe by mounting them on a thin substrate and using as high a beam voltage as possible or by mounting them on a normal substrate and using as low a beam voltage as possible? Will there be enough x-ray signal from the particles of interest and to what degree will the $\mathrm{x}$-ray spectrum be contaminated by signals from surrounding materials including other nearby particles and the substrate?

To help us decide what analytical conditions are correct for nanomaterials, we are using NISTMonte $[2,3]$ to model the interaction of the electron beam with two adjacent nanometer sized particles of different composition. The simulated particles are $\mathrm{Al}_{2} \mathrm{O}_{3}$ and $\mathrm{Fe}_{2} \mathrm{O}_{3}$ and vary in size from $10 \mathrm{~nm}$ to $500 \mathrm{~nm}$. The electron beam is $10 \mathrm{~nm}$ in diameter and is offset from the center of one particle to the center of the other particle in 10 steps. A schematic diagram of the simulation model is given in Figure 1. The accelerating voltage was simulated at $5 \mathrm{kV}$ and $25 \mathrm{kV}$ to represent the extremes of the voltages commonly available on commercial instruments. Experimental data collected under similar conditions on the FEI Quanta $200 \mathrm{~F}$ environmental scanning electron microscope [4] is presented and contrasted with the NISTMonte simulated results. An example of one data simulation is displayed in Figure 2. These results are from a 10,000 electron simulation of the electron beam in position 10 (Figure 1) on the center for the iron oxide particle. In this example, the particles are 100 $\mathrm{nm}$ in diameter and the $10 \mathrm{~nm}$ Gaussian profile electron beam is at $25 \mathrm{kV}$ potential. Figure $2 \mathrm{~A}$ shows the Fe x-rays generated by the beam and Figure $2 \mathrm{~B}$ shows the parasitic $\mathrm{Al} \mathrm{x}$-rays generated by electrons scattered from the $\mathrm{Fe}_{2} \mathrm{O}_{3}$ nanoparticle. The carbon x-rays from the substrate are displayed in Figure $2 \mathrm{C}$. The traces in Figure 2D represent the first 100 electrons paths (light blue in the particle, dark blue escaped into the vacuum, green thru the vacuum and into the substrate, and gray in the substrate material).

\section{References}

[1] J.A. Small et al., Handbook of X-Ray Spectrometry Revised and Expanded (Practical

Spectroscopy, V. 29, eds. R.E. Van Grieken and A.A. Markowicz, Dekker, New York, 2002, 811-926. 
[2] NISTMonte is written by Nicholas W.M. Ritchie and is distributed freely by NIST at http://www.cstl.nist.gov/div837/837.02/epq/index.html.

[3] J.A. Small and N.M.W. Ritchie, Microsc. Microanal. 11(Suppl 2) (2005) 1340.

[4] Certain commercial equipment, instruments, or materials are identified in this report to specify adequately the experimental procedure. Such identification does not imply recommendation or endorsement by the National Institute of Standards and Technology, nor does it imply that the materials or equipment identified are necessarily the best available for the purpose.

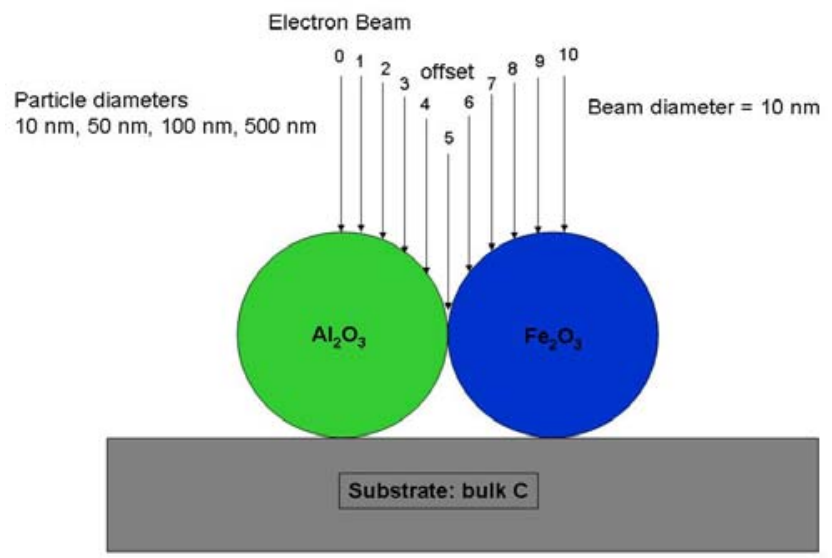

FIG. 1. Schematic diagram for the set up of the electron scattering simulation with NISTMonte with two dissimilar particles of the same but varied size and a beam impact spot that moves in ten equal steps from the center of one particle to the center of the other particle.
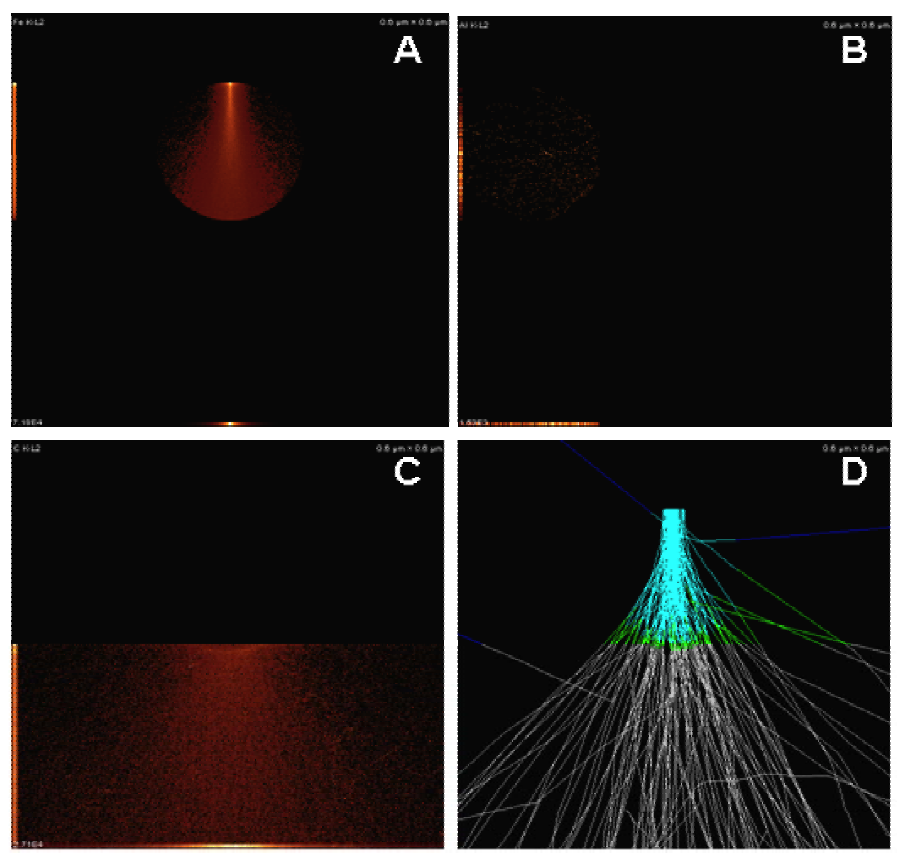

FIG. 2. Results of the NISTMonte simulation; A) Fe x-rays from the electron beam on the $100 \mathrm{~nm}$ iron oxide particle at $25 \mathrm{keV}, \mathrm{B}) \mathrm{Al}$ x-rays from scattered electrons, C) Carbon x-rays from substrate, D) Scatter diagram. 